\title{
BMJ Open Implementation and dissemination of home and community-based interventions for informal caregivers of people living with dementia: a systematic scoping review protocol
}

\author{
Eden Meng Zhu (D) , ${ }^{1}$ Martina Buljac-Samardžić, ${ }^{1}$ Kees Ahaus, ${ }^{1}$ Nick Sevdalis, ${ }^{2}$ \\ Robbert Huijsman ${ }^{1}$
}

To cite: Zhu EM, Buljac-

Samardžić M, Ahaus K, et al. Implementation and dissemination of home and community-based interventions for informal caregivers of people living with dementia: a systematic scoping review protocol. BMJ Open 2022;12:e052324. doi:10.1136/ bmjopen-2021-052324

- Prepublication history and additional supplemental material for this paper are available online. To view these files, please visit the journal online (http://dx.doi.org/10.1136/ bmjopen-2021-052324).

Received 15 April 2021 Accepted 06 January 2022

Check for updates

(C) Author(s) (or their employer(s)) 2022. Re-use permitted under CC BY-NC. No commercial re-use. See rights and permissions. Published by BMJ.

${ }^{1}$ Erasmus School of Health Policy and Management, Erasmus University Rotterdam, Rotterdam, The Netherlands

${ }^{2}$ Centre for Implementation

Science, King's College London, London, UK

Correspondence to

Eden Meng Zhu;

zhu@eshpm.eur.nl

\section{ABSTRACT}

Introduction Ageing in place, supported by formal home and community services and informal caregivers, is the most used long-term care option for people with dementia (PwD). Informal caregivers are inundated by their caregiving responsibilities and resultantly suffer consequences. Despite the multitude of clinical effectiveness studies on interventions that support informal caregivers, there is a paucity of information regarding their implementation process. This scoping review aims to identify the implementation strategies, implementation outcomes, and barriers and facilitators that impede or support the dissemination and uptake of interventions that support informal caregivers of PwD at home.

Methods and analysis This protocol is guided by the Preferred Reporting Items for Systematic Review and Meta-Analysis (PRISMA) Protocols, and the scoping review will follow the systematic steps of the PRISMA-Extension for Scoping Reviews guideline. The search strategy will include publications produced from inception to 8 March 2021 and will be conducted in the search engines Embase, Medline (Ovid), Web of Science and Cochrane Central Register of Controlled Trials (Wiley), followed by a three-stage approach. First, title and abstracts will be screened by two independent reviewers. Second, full-text articles will also be screened by both reviewers and, in case of disagreement, by a third reviewer. The first two stages are based on a set of inclusion and exclusion criteria. Reference lists of the final included studies will also be checked for relevant articles. Data from the final included studies will be extracted and synthesised using the Expert Recommendations for Implementing Change compilation and Proctor's implementation outcomes to ensure homogenous and standardised reporting of implementation information.

Ethics and dissemination The review findings will be published in a peer-reviewed journal and disseminated at geriatric and implementation conferences to inform researchers, health service planners and practice professionals with an overview of the existing literature to guide them in the effective implementation of caregiverfocused interventions in dementia support.

\section{Strengths and limitations of this study}

- This will be the first scoping review focused on studies that directly report implementation and dissemination of a full range of home and community-based interventions for informal caregivers of people with dementia (PwD).

- The findings from this review will provide synthesised evidence that guides implementation of the overwhelming number of clinical effectiveness studies of interventions for informal caregivers for PwD and provide insight into the link between intervention studies and implementation studies, promoting the dissemination and uptake of contextually appropriate interventions

- This will be one of the first reviews that uses the data management software of ASReview, as an Artificial intelligence-aided tool for title and abstract screening, promoting the integrated use of an open source artificial intelligence programme to systematically review extensive amounts of literature and to improve researcher efficiency without risking the review integrity.

- As it is a scoping review, the quality of included studies will not be formally assessed.

\section{INTRODUCTION}

Dementia is a neurocognitive disorder that affects over 36 million people and is expected to physically affect 66 million by 2030. ${ }^{1}$ People with dementia (PwD) gradually become incapable of independent living and lose the capacity to independently make informed decisions. They require extensive care provided by caregivers throughout the remainder of their lives, often within a formal care institution (eg, nursing home, long-term residential care facility). ${ }^{2}$ Previous studies have indicated that PwD prefer home-based care with support from formal and informal caregivers. ${ }^{3}$ Informal caregivers are identified 
as any individual who provides 'unpaid care to older and dependent persons with whom they have a social relationship, such as a spouse, parent, child, other relative, neighbour, friend or non-kin'. ${ }^{45}$ For those at more advanced stages of dementia, regular support from informal caregivers is essential to maintaining activities of daily living. As the global prevalence of dementia cases increases, more spouses and children of $\mathrm{PwD}$ will adopt the role of the primary informal caregiver and become inundated with responsibilities.

Resultantly, the quality of life for informal caregivers of PwD has become a global issue. ${ }^{6}$ Studies conducted across Europe found that informal caregivers often indicated a need for formal care for their relatives with dementia due to the impacted quality of life they experience in their role, the difficulties with managing behavioural problems of $\mathrm{PwD}$ and the limited access to effective community-based respite and supportive care services. ${ }^{7}$ In response, researchers and health policy actors have explored opportunities to develop and implement community-based interventions for informal caregivers of $\mathrm{PwD}$ that support and encourage the delivery of long-term care at home, or ageing in place, and delay institutionalisation. In the UK, 'Living well with Dementia' is a top priority in the national dementia strategy, which includes the development and implementation of supportive services for caregivers of PwD living at home. ${ }^{8}$ In the Netherlands, the Ministry of Health, Welfare and Sport ${ }^{9}$ recently published The National Dementia Strategy 2021-2030, which reported an estimated national figure of 350000 informal caregivers for $\mathrm{PwD}$, within a total national population of 17 million persons, $31 \%$ of whom devote more than 40 hours per week to providing informal care. The growing focus towards improving support for informal caregivers accelerates the development and implementation of more evidence-based programmes that support and sustain home-based and community-based care.

Furthermore, Wübker et $a l^{10}$ reported that the average monthly cost of institutionalised and professional homebased long-term care for PwD across eight European countries amounted to 4491 Euro and 2491 Euro, respectively. These results reveal the magnitude of the demand for dementia care providers, the relatively high costs of institutionalised care and the value of supplemental formal home-based and community-based dementia care resources. Previous studies have also indicated that PwD personally prefer to receive delay institutionalisation and receive care at home due to their desire to maintain autonomy and preserve their personhood. ${ }^{11}$ Informal caregivers of $\mathrm{PwD}$ have also previously associated institutionalisation with abandonment and mainly considered this option once the disorder progressed and presented unmanageable complex care demands or once their resources became limited or insufficient to sustain homebased care. ${ }^{12}$ Additionally, informal care for PwD living at home is the only feasible option in resource-limited countries. ${ }^{13}$
In response to this demand, health policy actors are urged to invest in developing and implementing sustainable home and community-based care solutions for $\mathrm{PwD}$ and their informal caregivers that delay or replace institutionalisation to conserve economic resources and to satisfy the preferences of $\mathrm{PwD}$ and their informal caregivers. Given these conditions, the self-efficacy and caregiving competencies of informal caregivers ultimately determine care outcomes for $\mathrm{PwD}$ and informal caregivers; proper education, support and resources provided by formal care providers are essential to support informal caregivers in their role. ${ }^{14}$ Without adequate support, according to the stress process theory, informal caregivers are more vulnerable to developing depression and anxiety and become more susceptible to developing chronic illnesses exacerbated by stress and, subsequently, compromising their caregiving abilities. ${ }^{15} 16$

\section{Rationale for review}

The implementation process of interventions that support informal caregivers of PwD must be examined in addition to intervention studies to gain a comprehensive understanding of their usability and real-world value and impact. ${ }^{17}$ As for effectiveness studies of interventions, Cheng and Zhang $^{18}$ recently published a meta-review that included 60 separate review articles, amalgamating over 500 individual articles that examined the effectiveness of various informal caregiver-focused interventions. They identified the main types of interventions available for informal caregivers of $\mathrm{PwD}$, including psychoeducation and psychotherapy (eg, cognitive behavioural therapy), support groups, respite care, caregiver training (eg, occupational training) and mindfulness and exercise programmes. ${ }^{13}$ However, previous studies have often reported a need for additional implementation studies that report strategies to 'translate caregiver interventions into practice' and 'evaluate the mechanisms for sustainability within the healthcare system' ${ }^{19}{ }^{20}$ Successful implementation also requires a comprehensive understanding of the barriers and facilitators to implementation and the contextual factors influencing dissemination of evidencebased practices. ${ }^{17}$

In light of this evidence, this review is grounded onto theory and concepts developed within the recently merged multidisciplinary field of implementation science. Implementation science seeks to understand and characterise the process of translating evidence into routine practice in healthcare settings, with the ultimate aim of accelerating this translation and ensuring healthcare practice is consistently and appropriately evidence based. ${ }^{21}$ In doing so, the field has developed a clear focus on so-called 'implementation strategies', defined as methods or techniques used to support and enhance the adoption, implementation and sustainability of an intervention clinical intervention. ${ }^{22}$ The most comprehensive mapping of such implementation support interventions was developed in the context of the Expert Recommendations for Implementing Change (ERIC) study. ${ }^{22}$ Following 
literature review and an expert consensus process, ERIC developed a compilation of 73 implementation strategies that has allowed researchers to report implementation process details using a homogenous and consistent approach. Waltz et $a l^{23}$ further compiled the 73 strategies into nine thematic clusters, including evaluative and iterative strategies, provide interactive assistance, adapt and tailor to context, develop stakeholder interrelationships, train and educate stakeholders, support clinicians, engage consumers, use financial strategies and change infrastructure. These clusters will provide one part of the conceptual framework for this review. The other part of this framework will be offered by a brief taxonomy of 'implementation outcomes', defined as the effects of deliberate and purposive actions to implement new treatments or services. ${ }^{24}$ The most established taxonomy for these outcomes has been developed by Proctor et $a l^{24}$ who identified acceptability, adoption, appropriateness, feasibility, fidelity, cost, penetration and sustainability as a core set of implementation outcomes to be measured and studies alongside patient and service-level outcomes. The corpus of evidence that this review will identify will be synthesised through the prism of implementation strategies and outcomes.

To-date, a few reviews have focused on implementation strategies in the area of dementia care. Lourida $e t$ $a l^{25}$ presented a scoping review of implementation and dissemination strategies of interventions for the dementia care recipient (ie, PwD). Bennet et $a l^{26}$ also published a systematic review on implementation studies of nonpharmacological interventions addressing behavioural and psychological symptoms of dementia. Although the reviews of Lourida et $a l^{25}$ and Bennett et $a l^{26}$ do focus on implementation strategies, interventions were not focused on informal caregivers. The review of Christie et $a l^{27}$ did focus on implementation strategy of interventions for informal caregivers of $\mathrm{PwD}$ living at home; however, they limited their focus to eHealth interventions and excluded implementation studies on the various other types of interventions available to support informal caregivers in their role. Furthermore, the UK National Institute for Health Research ${ }^{28}$ and the Dutch Research Council ${ }^{29}$ have both released calls for research proposals focusing primarily on supporting PwD and their informal caregivers carers and enhancing their quality of life. Based on these findings, this study aims to produce a scoping review to synthesise the available evidence relating to the implementation of interventions that support informal caregivers of PwD.

\section{Review aim and objectives}

The aim of the scoping review is to provide an overview of reported implementation insights of interventions for informal caregivers of PwD living at home. Our specific objectives are to identify the implementation strategies, implementation outcomes and barriers and facilitators that impede or support the dissemination and uptake of interventions. All three objectives are essential to developing a comprehensive review that will sufficiently inform the development of future interventions and their implementation plans without creating further information fragmentation.

\section{METHODS}

Scoping review methodology with a systematic search strategy will be applied to this review. According to Arksey and O'Malley, ${ }^{30}$ a scoping review is most suitable to summarise the range of evidence, to disseminate the research findings and to expose information gaps in the existing literature; scoping reviews also cover broader topics presented through various study designs. The proposed scoping review is guided by a five-step framework by Arksey and O'Malley, ${ }^{30}$ which includes (1) identifying research questions, (2) constructing a primary search strategy and (3) identifying and selecting relevant studies with a clear inclusion and exclusion criterion, (4) extracting and charting the relevant data and (5) summarising, collating and reporting the final results. This protocol was guided by the PRISMA-P (Preferred Reporting Items for Systematic Review and Meta-Analysis Protocols) (see online supplemental file 1: PRISMA-P) ${ }^{31}$ A brief protocol for this review has also been registered in the Open Science Framework (osf.io/tvdb5) to provide transparency throughout the review process. ${ }^{32}$ The final scoping review will follow thePRISMA-Extension for Scoping Reviews (see online supplemental figure 1: PRISMA-ScR) ${ }^{33}$

\section{Identifying the research questions}

The main research question is 'what are the implementation and dissemination strategies reported for home and community-based interventions that support the informal caregivers of people with dementia living at home?'. Three subquestions were developed that will lead to answering the main question:

1. What implementation strategies have been reported for interventions that support informal caregivers of people with dementia living at home?

2. What are the implementation outcomes reported for these interventions?

3. What are the reported barriers and facilitators of implementation and dissemination that impede or support the uptake and utilisation of these interventions?

\section{Search strategy}

First, a limited search of EMBASE and MEDLINE was conducted to identify articles focusing on interventions for caregivers of PwD; texts that fit the search domain were analysed to determine key index terms. Following, with additional support from a medical librarian, an initial search strategy comprised of the identified key terms relating to 'dementia', 'informal caregivers', 'intervention' and 'implementation and dissemination' was developed (see online supplemental file 3: Full Search Strategy). Articles published from inception through 
8 March 2021 will be included. The search strategy will be adapted for use in Embase, Medline (Ovid), Web of Science and Cochrane Central Register of Controlled trials (Wiley) to ensure comprehensive literature in the final search outcomes. Results obtained across these databases will be compiled and deduplicated prior to screening.

\section{Identifying and selecting relevant studies}

The full process of identifying and selecting relevant studies will have three stages. First, the titles and abstracts of all unique results previous obtained will be imported and screened manually by two independent reviewers (EMZ and MB-S) using the novel ASReview tool (https:/ / asreview.nl/). ${ }^{34}$ According to van de Schoot $e t a \hat{l}^{34}$ ASReview is able to detect $95 \%$ of the eligible studies after screening between only $8 \%$ to $33 \%$ of the studies, which significantly reduces time spent screening titles and abstracts. Ferdinands ${ }^{35}$ applied ASReview to a full set of 5050 studies that were previously manually identified and screened by another review to evaluate the tool's operational performance. The results revealed that ASReview was also able to obtain 'more than $80 \%$ of relevant publications after screening only $10 \%$ of all publications' and 'identified $95 \%$ of relevant publications after screening about $20 \%$ of all publications', thus reducing screening effort by $78 \%-82 \% .{ }^{35}$ ASReview was selected as a screening tool due to its novel use of machine learning to first find and present the titles and abstracts in an efficient order, from most relevant to least relevant, which will allow the reviewers to manually filter all results quickly and efficiently without compromising the review's integrity. ${ }^{34}$

The title and abstract screening process will use a twopronged approach. The first reviewer (EMZ) will manually screen all of the title and abstracts using ASReview and includes and exclude studies based on the exclusion criteria. The full text of included studies by the first reviewer will be screened in the next stage. Following, using ASReview, the second reviewer (MB-S) will manually review all of the studies excluded by the first reviewer to ensure that all relevant studies have been considered for full-text assessment; once 50 successive articles have been excluded, the second reviewer will stop screening. The full texts of all studies included by the second reviewer will also be assessed to avoid any false negatives.

Second, the selected studies will undergo a full-text evaluation, conducted by two independent reviewers (EMZ and MB-S), who closely examine the population, intervention and outcomes reported in the studies to determine if the study is suitable for the purpose of this review and to avoid false positives obtained in the first step. If there are any disagreements at this stage, a third reviewer will read the full text and discuss the areas of contention with the two independent reviewers to reach a consensus. Third, included articles will undergo a reference list check to ensure that relevant articles are found in this scoping review. ProQuest RefWorks (https://refworks. proquest.com) will be used to manage full-text articles and citations. ${ }^{36}$ The screening process and reasons for exclusion will be reported using the PRISMA flow diagram (see online supplemental figure 2: PRISMA-ScR). ${ }^{33}$

In accordance with Arksey and O'Malley's scoping review methodology and reporting guideline, ${ }^{30}$ the inclusion and exclusion criteria may be iteratively refined during the review process; any modifications made in the full scoping review will be reported. This review will consider all empirical studies published in peer-reviewed journals to ensure veracity of information; it will exclude any type of systematic reviews, book chapters, editorial letters, opinion papers or grey literature. There are no limitations on the types of interventions included, but they must directly aim to impact the informal caregiver of PwD. Literatures published in languages other than English are excluded due to resource limitations. Study should focus on the implementation and dissemination of interventions for informal caregivers of PwD living at home; for example, spouses, children, neighbours or friends. All types of interventions are included in this review if they directly support or impact the informal caregiver of PwD living at home.

To be included in this review, studies must either: (1) explicitly report detailed information on implementation strategies used and implementation outcomes examined for all types of evidence-based interventions, delivered at home or within the community, that directly impact the experience of informal caregivers of PwD living at home or (2) present detailed information on the perceptions and attitudes, or barriers and facilitators, involved in the implementation and dissemination process of these interventions from the informal caregiver perspective. This review will exclude all studies that present interventions delivered within formal institutional care settings or have a primary focus on formal care providers as study participants. Dyadic interventions that provide care for $\mathrm{PwD}$, without direct impact on the informal caregiver, will also be excluded. Studies that involve interventions for informal caregivers of people with conditions other than dementia will also be excluded.

\section{Data charting}

Data from the included studies will be initially extracted using a data extraction table that includes study characteristics, including first author, year of publication, country, study design and frameworks used, aim and purpose of study, types of intervention as reported in Cheng $e t a l^{18}$ participant details (eg, number of participants, relationship between informal caregiver and $\mathrm{PwD}$ ) and main outcomes reported within the included study.

An initial selection of 10 selected studies will be used as a pilot sample. One reviewer will extract data from this sample and populate the extraction form. The second reviewer will assess the accuracy and suitability of the domains analysed based on the study's objectives; disagreements between two reviewers will be resolved within the team. The data from the remaining included studies will then be extracted by the first author using the 
refined data extraction table. Any iterative modifications made to the data extraction table will be reported in the full scoping review article.

\section{Collating, summarising and reporting the results}

The main outcomes from this review will build on implementation science literature and use the 73 implementation strategies identified through the ERIC study ${ }^{22}$ and the nine thematic clusters identified in Waltz et $a l^{23}$ to structure and homogenise the reporting of implementation data obtained through the included studies. Furthermore, reported implementation outcomes within these included articles will be extracted and structured with guidance from evidence provided in Proctor $e t ~ a l .^{24}$ The focus on these two aspects will allow researchers to synthesise implementation evidence from interventions across various contexts. This review will also include the identified barriers and facilitators to implementation and dissemination, including organisational, professional, individual, financial and other perspectives, to gain a comprehensive understanding of the contextual factors that influence outcomes.

\section{Patient and public involvement}

A primary aim of this review is to offer an overview of what appears currently to be a rather disparate evidence base and to use formal implementation science concepts to synthesise and organise this evidence. People with dementia, formal or informal caregivers or healthcare professionals working in dementia services will be involved in the stages following the review publication. For example, a follow-up empirical study will validate the scoping review's findings and explore end users' perspectives on what might be viable and desirable approaches to tailor the implementation and dissemination of support interventions identified and/or to address the barriers to their scale-up application in support of informal caregivers. End users will not be involved in any phase of the review work. The first phase in which end users will be involved is when a viable and shareable summary of the review will be distributed.

\section{RESULTS}

Findings will be extracted and reported using a narrative synthesis approach to determine the key contextual determinants influencing the implementation and uptake process as well as the reported data regarding the implementation of caregiver-focused interventions to clarify the gaps that require further resource commitment and research. The results will also reveal the nature and trend of the existing literature in implementation science regarding informal caregiver interventions and explore how implementation is being reported to contribute to a more standardised homogenous reporting strategy.

\section{Ethics and dissemination}

This scoping review aims to guide the direction of future research towards the evidence-driven implementation of effective, evidence-based practices that support informal caregivers of people living with dementia at home. The review will not require ethical approval since it will not involve fresh primary data collection, and the findings will be published in a peer-reviewed journal and disseminated at future conferences on geriatric care and implementation science.

Online supplemental figure 1 presents the PRISMA-ScR, which will be used to guide the reporting of the final scoping review. Online supplemental figure 2 presents the PRISMA flow diagram, which will provide transparency during the text screening and final inclusion and exclusion process in the final scoping review.

Acknowledgements The authors wish to thank Dr Sabrina Meertens-Gunput from the Erasmus MC Medical Library for developing and updating the search strategies and Dr Rens van de Schoot from Utrecht University for his consultation on the operationalisation of ASReview for title and abstract screening.

Contributors EMZ and MB-S formulated the idea, and developed the research questions and the methodology of this review and review protocol. KA, RH and NS contributed to designing the research methods. EMZ drafted the manuscript, and all authors contributed the editing and approval of the final manuscript.

Funding The authors have not declared a specific grant for this research from any funding agency in the public, commercial or not-for-profit sectors.

Competing interests NS is the director of the London Safety and Training Solutions Ltd, which offers training in patient safety, implementation solutions and human factors to healthcare organisations and the pharmaceutical industry. The other authors have no conflicts of interest to declare.

Patient consent for publication Not applicable.

Provenance and peer review Not commissioned; externally peer reviewed.

Supplemental material This content has been supplied by the author(s). It has not been vetted by BMJ Publishing Group Limited (BMJ) and may not have been peer-reviewed. Any opinions or recommendations discussed are solely those of the author(s) and are not endorsed by BMJ. BMJ disclaims all liability and responsibility arising from any reliance placed on the content. Where the content includes any translated material, BMJ does not warrant the accuracy and reliability of the translations (including but not limited to local regulations, clinical guidelines, terminology, drug names and drug dosages), and is not responsible for any error and/or omissions arising from translation and adaptation or otherwise.

Open access This is an open access article distributed in accordance with the Creative Commons Attribution Non Commercial (CC BY-NC 4.0) license, which permits others to distribute, remix, adapt, build upon this work non-commercially, and license their derivative works on different terms, provided the original work is properly cited, appropriate credit is given, any changes made indicated, and the use is non-commercial. See: http://creativecommons.org/licenses/by-nc/4.0/.

ORCID iD

Eden Meng Zhu http://orcid.org/0000-0001-5039-0578

\section{REFERENCES}

1 Prince M, Guerchet M, Prina M. The global impact of dementia 20132050. London: Alzheimer's Disease International, 2013.

2 Hegde S, Ellajosyula R. Capacity issues and decision-making in dementia. Ann Indian Acad Neurol 2016;19:34-9.

3 Chester H, Clarkson P, Davies L, et al. People with dementia and carer preferences for home support services in early-stage dementia. Aging Ment Health 2018;22:270-9.

4 Broese van Groenou MI, De Boer A. Providing informal care in a changing society. Eur J Ageing 2016;13:271-9.

5 Triantafillou Jet al. Informal care in the long-term care system: European overview paper. Athens/Vienna: Interlinks, 2010. 
6 Brodaty H, Donkin M. Family caregivers of people with dementia. Dialogues Clin Neurosci 2009;11:217-28.

7 Vandepitte S, Putman K, Van Den Noortgate N, et al. Factors Associated with the Caregivers' Desire to Institutionalize Persons with Dementia: A Cross-Sectional Study. Dement Geriatr Cogn Disord 2019;46:298-309.

8 Banerjee S. Living well with dementia-development of the National dementia strategy for England. Int J Geriatr Psychiatry 2010;25:917-22

9 Ministry of Health, Welfare and Sport, Netherlands. National dementia strategy 2021-2030. Ministry of Health, Welfare and Sport, 2020: 2-23.

10 Wübker A, Zwakhalen SMG, Challis D, et al. Costs of care for people with dementia just before and after nursing home placement: primary data from eight European countries. Eur $\mathrm{J}$ Health Econ 2015;16:689-707.

11 Smebye KL, Kirkevold M, Engedal K. Ethical dilemmas concerning autonomy when persons with dementia wish to live at home: a qualitative, hermeneutic study. BMC Health Serv Res 2015;16.

12 Nunez FE. Factors influencing decisions to admit family members with dementia to long-term care facilities. Nurs Forum 2021;56:372-81.

13 Goodson M, McLellan E, Rosli R, et al. A qualitative study on formal and informal carers' perceptions of dementia care provision and management in Malaysia. Front Public Health 2021;9:637484.

14 Chiao C-Y, Wu H-S, Hsiao C-Y. Caregiver burden for informal caregivers of patients with dementia: a systematic review. Int Nurs Rev 2015;62:340-50.

15 Kaddour L, Kishita N. Anxiety in informal dementia carers: a metaanalysis of prevalence. J Geriatr Psychiatry Neurol 2020;33:161-72.

16 Alves LCdeS, Bento SR, Bento SR, et al. Burnout syndrome in informal caregivers of older adults with dementia: a systematic review. Dement Neuropsychol 2019;13:415-21.

17 Bauer MS, Kirchner J. Implementation science: what is it and why should I care? Psychiatry Res 2020;283:112376.

18 Cheng S-T, Zhang F. A comprehensive meta-review of systematic reviews and meta-analyses on nonpharmacological interventions for informal dementia caregivers. BMC Geriatr 2020;20:137.

19 Elvish R, Lever S-J, Johnstone J, et al. Psychological interventions for carers of people with dementia: a systematic review of quantitative and qualitative evidence. Couns Psychother Res 2013:13:106-25.

20 Piersol CV, Canton K, Connor SE, et al. Effectiveness of interventions for caregivers of people with Alzheimer's disease and related major neurocognitive disorders: a systematic review. Am J Occup Ther 2017;71:7105180020p1-7105180020.

21 Nilsen P. Making sense of implementation theories, models and frameworks. Implement Sci 2015;10:53.
22 Powell BJ, Waltz TJ, Chinman MJ, et al. A refined compilation of implementation strategies: results from the expert recommendations for implementing change (ERIC) project. Implement Sci 2015;10:21.

23 Waltz TJ, Powell BJ, Matthieu MM, et al. Use of concept mapping to characterize relationships among implementation strategies and assess their feasibility and importance: results from the expert recommendations for implementing change (ERIC) study. Implement Sci 2015;10:109.

24 Proctor E, Silmere H, Raghavan R, et al. Outcomes for implementation research: conceptual distinctions, measurement challenges, and research agenda. Adm Policy Ment Health 2011;38:65-76.

25 Lourida I, Abbott RA, Rogers M, et al. Dissemination and implementation research in dementia care: a systematic scoping review and evidence MAP. BMC Geriatr 2017:17:147.

26 Bennett S, Laver K, MacAndrew M, et al. Implementation of evidence-based, non-pharmacological interventions addressing behavior and psychological symptoms of dementia: a systematic review focused on implementation strategies. Int Psychogeriatr 2021;33:1-29.

27 Christie HL, Bartels SL, Boots LMM, et al. A systematic review on the implementation of eHealth interventions for informal caregivers of people with dementia. Internet Interv 2018:13:51-9.

28 NIHR. 21/16 improving support for adult and/or older carers, 2021. Available: https://www.nihr.ac.uk/funding/2116-improving-supportfor-adult-andor-older-carers/27123 [Accessed Mar 2021].

29 NWO. Living with dementia, 2021. Available: https://www.nwo.nl/en/ calls/living-dementia [Accessed Mar 2021].

30 Arksey H, O'Malley L. Scoping studies: towards a methodological framework. Int J Soc Res Methodol 2005;8:19-32.

31 Moher D, Shamseer L, Clarke M, et al. Preferred reporting items for systematic review and meta-analysis protocols (PRISMA-P) 2015 statement. Syst Rev 2015;4:1.

32 Zhu E, Buljac-Samardžić M, Ahaus K. Implementation and dissemination of home and community-based interventions for informal caregivers of people living with dementia: a systematic scoping review protocol 2021

33 Tricco AC, Lillie E, Zarin W, et al. PRISMA extension for scoping reviews (PRISMA-ScR): checklist and explanation. Ann Intern Med 2018:169:467-73.

34 van de Schoot R, de Bruin J, Schram R, et al. An open source machine learning framework for efficient and transparent systematic reviews. Nat Mach Intell 2021;3:125-33.

35 Ferdinands G. Al-Assisted systematic reviewing: selecting studies to compare Bayesian versus Frequentist SEM for small sample sizes. Multivariate Behav Res 2021;56:153-4.

36 Ivey C, Crum J. Choosing the right citation management tool: endnote, Mendeley, RefWorks, or Zotero. Jmla 2018;106:399-403. 